\title{
Öber Pankreassteapsin und über die Reaktionsgeschwindigkeit der mittels Enzyme bewirkten Fettspaltung.
}

\author{
Von
}

Aristides Kanitz.

(Der Redaktion zugegangen am 25. November 1905.)

Ob zwar schon M. Nencki's 1886 veröffentlichte Versuche die energische, esterspaltende Eigenschaft der zerkleinerten Pankreasdrüse in überzeugender Weise dartun ${ }^{1}$ ) und seither von mehreren Firmen wirksame Steapsinpräparate in Handel gebracht wurden, ${ }^{2}$ ) sind immerhin die Mitteilungen über die fettspaltende Fähigkeit von Pankreasauszügen, wie man u. a. aus W. Connsteins Monographie «Über fermentative Fettspaltung ${ }^{3}$ ) entnehmen kann, recht widerspruchsvoll.

1) M. Nencki, Über die Spaltung der Säureester der Fettreihe und der aromatischen Verbindungen im Organismus und durch das Pankreas. Arch. f. experim. Path. u. Pharm., Bd. XX, S. 367-384, 1886.

2) Die Glycerinauszüge des von der chem. Fabrik RhenaniaAachen hergestellten «Pankreatin absol.» sollen eine Eigelbemulsion gut spalten. Dr. G. Grübler in Dresden hat mir eine wirksame «Steapsinsolution» geliefert. schienen.

3) In den Ergebn. der Physiol., Bd. III, Abt. 1, S. 194-232, 1904, er-

Den kritischen Ausführungen des Autors kann zugestimmt werden.

Unter den vielen Einwendungen, welche gegen Hanriot's Arbeiten über Lipase gemacht worden sind, hat zufälligerweise niemand darauf hingewiesen, daß seine sämtlichen Bestimmungen (und auch viele seiner Gegner) mit einem unkontrollierbaren Fehler behaftet sind, indem er die aus dem Monobutyrin abgespaltene Buttersäure mit Soda und Phenolphtaleïn als Indikator titriert. Das ist zweifach unrichtig. Erstens ist, um die Hydrolyse zu vermeiden, eine $\mathrm{schwach}$ dissoziierte Säure, wie die Buttersäure es ist, mit einer stark dissoziierten Base (Kalilauge, Baryumhydroxyd) zu titrieren. Zweitens kann Phenolphtaleïn wegen seiner äußerst schwach sauren Eigenschaft in Gegenwart von Kohlensäure nicht als Indikator verwendet werden. 
Von den Autoren, die in den letzten Jahren über den Gegenstand gearbeitet haben, berichten Kastle und Loewenhart, $\left.{ }^{1}\right)$ daß ihre Auszüge, welche Äthylbutyrat gut gespaltet haben, Neutralfette kaum zerlegen konnten. Hans Engel ${ }^{2}$ ) gelang es nur einmal, aus der Drüse einen wirksamen Auszug zu erhalten, sodaß er ein "Zeit- und Fermentgesetz» für das Pankreassteapsin nur an der Hand von Versuchen, welche mit einem Handelspräparat ausgeführt waren, aufstellen konnte. J. Lewkowitsch und J. J. R. Macleod ${ }^{3}$ ) berichten zwar, daß sie neutralfettspaltende, wässerige Auszüge erhalten haben - die Zeit jedoch, welche zur erheblichen Spaltung des verwendeten Baumwollsamenöls erforderlich war, beläuft sich auf Monate.

Dabei wäre ein jeder Versuch, in welchem mittels Pankreasauszügen oder Pankreassuspensionen binnen wenigen Stunden eine erhebliche Fettspaltung erzielt wird, ein weiterer Beweis zugunsten der Theorie, daß die Fette als Fettsäuren resorbiert werden!

Nach verschiedenen mißlungenen Versuchsanordnungen habe ich durch oft wiederholte Versuchsreihen gefunden, daß man - im Gegensatz zu der verbreiteten Ansicht über die große Empfindlichkeit des Steapsins - aus der Pankreasdrüse mit Glycerin in sehr einfacher Weise Auszüge herstellen kann, welche, bei geeigneter Versuchsanordnung, auch in geringer Menge angewandt, in kurzer Zeit erhebliche Neutralfettmengen spalten können.

Ich habe die vom hiesigen Schlachthof gelieferten Rindund Schweinpankreasdrüsen, welche etwa 24 Stunden, nachdem die Tiere geschlachtet waren, in meinen Besitz gelangten, von dem Fett möglichst befreit, mit einer Fleischhackmaschine zerkleinert, mit 2-3 facher Menge käuflichem Glycerin gut vermischt und ohne jede weitere Vorsichtsmaßregel mehrere

1) Amer. Chem. Journ., Bd. XXIV, S. 471, 1901.

2) Hans Engel, Über das Zeit- und Fermentgesetz des Pankreassteapsins. Hofmeister's Beiträge, Bd. VII, S. 77-88, 1905.

s) J. Lewkowitseh und J. J. R. Macleod, The Hydrolysis of Fats in vitro by means of Steapsin. Proc. Roy. Soc., Bd. LXXII, S. 31, 1903. 
Wochen bis Monate bei Zimmertemperatur gehalten, sodann durch Tücher koliert.

Zur Ermittlung der abgespaltenen Fettsäure habe ich das Spaltungsgemisch in genügend Alkohol und etwas Äther gelöst und mit wässeriger n- bezw. n/10-Natron-, später Kalilauge und Phenolphtaleïn als Indikator titriert, unter Einhaltung der Mindestalkoholkonzentration, welche nach meiner früheren Mitteilung ${ }^{1}$ ) erforderlich ist, um die Hydrolyse vollständig zu umgehen.

Die Spaltungsversuche selbst wurden in folgender Weise ausgeführt: $\mathrm{Zu}$ je $10 \mathrm{ccm}$ käuflichem sogenannten Olivenöl oder zu Rizinusöl, welche so viel freie Fettsäure enthielten, daß zu der Neutralisation der genannten Menge $2-5 \mathrm{ccm}$ n/10-Lauge erforderlich waren, wurde diese Laugenmenge zugesetzt und durch Schütteln mit dem Öl gut vermischt. Es entsteht so eine sehr feinverteilte und dauerhafte Emulsion, zu welcher sodann die Enzymlösung $(1-2 \mathrm{ccm})$ hinzugefügt wurde.

Nachdem die Spaltung 4-6 Stunden vor sich gegangen war, wurde titriert und es ergab sich, daß zur Neutralisation der abgespaltenen Fettsäure ca. $100 \mathrm{ccm}$ n/10-Lauge notwendig war. Es ist somit etwa ein Drittel des Fettes zerlegt worden. Wurde eine Enzymlösung verwendet, welche vorher 10 Minuten bei $100^{\circ}$ gehalten worden war, so war innerhalb der Versuchsdauer keine Fettsäure abgespalten worden.

Bei frischen Auszügen (auch bei dem von Dr. Grübler mir gelieferten Präparat) war die Spaltung energischer, wenn vorher einige Tropfen n-Calciumchloridlösung zu der Emulsion zugefügt waren. Calciumacetat war wirkungslos. Man könnte hierin die Aktivierung eines Zymogens oder eines Proenzyms erblicken, doch ich möchte vorderhand diese Frage nicht weiter diskutieren. Bei frischen, wässerigen Pankreasauszügen sollen nach H. Pottevin ${ }^{2}$ ) sowohl Calciumchlorid wie Calciumacetat und auch noch verschiedene andere Salze spaltungsfördernd wirken.

Die Spaltung verläuft bei Zimmertemperatur und bei $56^{\circ}$

1) Aristides Kanitz, Beiträge zur Titration von hochmolekularen Fettsäuren. Ber. d. Deutsch. chem. Ges., Bd. XXXVI, S. 400-404, 1903.

2) H. Pottevin, Sur le mécanisme des actions lipolytiques. Compt. rend.; Bd. CXXXVI, S. 767, 1903. 
mit einer etwa gleichen Geschwindigkeit, bei $40^{\circ}$ ist die Spaltungsgeschwindigkeit rund das Anderthalbfache der bei Zimmertemperatur. Hätte der Vorgang sein Optimum bei $40^{\circ}$ und denselben Temperaturkoeffizienten, wie die chemischen Reaktionen - welcher Temperaturkoeffizient auch für manche biologische Vorgänge zuzutreffen scheint ${ }^{1}$ ) -, so müßte bei $40^{\circ}$ die Spaltungsgeschwindigkeit etwa das 4- bis 9 fache der Geschwindigkeit bei Zimmertemperatur erreicht haben. Wie man aus Hanriots Versuchen ${ }^{2}$ ) ersieht, scheint auch die Lipase einen ganz außergewöhnlich kleinen Temperaturkoeffizienten zu haben.

Wird die Enzymlösung durch gehärtetes Filtrierpapier filtriert, so hat das Filtrat seine Wirksamkeit nicht verloren. Doch ist das Filtrat wirkungslos, wenn man den Auszug durch unglasierte Tonzellen filtriert. Es wäre somit die von Conntein (l. c.) hervorgehobene Unlöslichkeit des Steapsins bestätigt, ich möchte jedoch daran erinnern, daß, ob zwar, wie Versuche von A. Fernbach ${ }^{3}$ ) zeigen, die Hefeinvertase die Chamberland-Kerze nicht passiert, andere Invertasen das tun $;^{4}$ ) es also bei den Enzymen in bezug ihrer Löslichkeit allmähliche Übergänge $\mathrm{zu}$ geben scheint.

Über den zeitlichen Verlauf der Spaltung teile ich aus vielen ähnlichen die folgende, mit einem sehr trägen Präparat bei $20^{\circ}$ gewonnene Tabelle mit.

Sie wurde erhalten, indem man in einer Anzahl Reagensgläser je $10 \mathrm{ccm}$ Olivenöl, $3,8 \mathrm{ccm} \mathrm{n/10-Natronlauge,} 0,25 \mathrm{ccm}$ n-Calciumchlorid und $1 \mathrm{ccm}$ Steapsin vermischte und den Inhalt je eines Reagenzglases nach der unter $\vartheta$ verzeichneten Minutenzahl titrierte. Die dabei verbrauchten Kubikzentimeter

1) R. A begg, Der Temperatureinfluß auf die Entwicklungsgeschwindigkeit animalischen Lebens. Zeitschr. f. Elektrochemie, Bd. XI, S. 529, 1905. - Aristides Kanitz, Über den Einfluß der Temperatur auf die Kohlendioxydassimilation. Ibid. Bd. XI, Heft 42, 1905.

2) Die betreffende Tabelle ist bei Connstein (l. c., S. 221) angeführt.

3) A. Fernbach, Sur le dosage de la sucrase. Ann. Inst. Pasteur, Bd. III, S. 531, 1889.

4) Wenn ich mich nicht irre, ebenfalls in der Fernbach'schen Arbeit erwähnt. 
n/10-Natronlauge sind unter $x$ gegeben. Unter $\frac{x}{g}$ ist die gespaltene Menge, dividiert durch die zugehörende Spaltungszeit, unter $\frac{x}{\sqrt{9}}$ die gespaltene Menge, dividiert durch die Quadratwurzel aus der Spaltungszeit, angeführt.

$\begin{array}{rccc}9 & \mathbf{x} & \frac{\mathrm{x}}{\mathrm{y}} & \frac{\mathrm{x}}{\mathbf{y}} \\ 0 & 0,0 & - & - \\ 70 & 9,2 & 0,131 & 1,10 \\ 140 & 12,3 & 0,088 & 1,04 \\ 288 & 19,0 & 0,065 & 1,12 \\ 405 & 23,1 & 0,057 & 1,15 \\ 1455 & 34,2 & 0,023 & 0,90\end{array}$

Während $\frac{x}{\vartheta}$ nicht konstant ist, ist das für $\frac{x}{\sqrt{y}}$ annähernd der Fall. Die Konstanz derselben Beziehung fand Engel (l. c.) bei der Spaltung von Eigelbemulsion mit Pankreatin-Rhenania. Ebenfalls dasselbe erhielt ich, als. ich die Versuchsdaten von W. Connstein, E. Hoyer und H. Wartenberg ${ }^{1}$ ) über den zeitlichen Verlauf der Spaltung des Ricinusöls mit dem im Ricinussamen enthaltenen Enzym daraufhin durchrechnete. Ich setze das Ergebnis als Fußnote her. ${ }^{2}$ )

Nach den Lehren der allgemeinen Chemie würden sich für die durch die Gegenwart eines Katalysators mit wahrnehm-

1) Über fermentative Fettspaltung. Ber. d. Deutsch. chem. Ges., Bd. XXXV, S. 3988-4006, 1903.

2) Versuch 46 (1. c., S. 4003). Von 6,5 g Ricinusöl waren in Gegenwart von $4 \mathrm{~g}$ n/10-Schwefelsäure nach 8 Minuten $x$ Prozente gespalten:

\begin{tabular}{rccc}
\multicolumn{9}{c}{} & $\mathrm{x}$ & $\frac{\mathrm{x}}{\mathrm{y}}$ & $\frac{\mathrm{x}}{\mathbf{y}}$ \\
15 & 12 & 0,80 & $\mathbf{4} 33$ \\
30 & 20 & 0,66 & $\mathbf{3}, 36$ \\
45 & 30 & 0,60 & $\mathbf{4}, 47$ \\
60 & 33 & 0,55 & 4,26 \\
90 & 41 & 0,45 & 4,32 \\
150 & 54 & 0,36 & $\mathbf{4}, 41$ \\
210 & 59 & 0,28 & $\mathbf{4}, 16$ \\
330 & 68 & 0,20 & 2,74 \\
1620 & 81 & 0,05 & 2,01
\end{tabular}


barer Geschwindigkeit verlaufende Fettspaltung folgende Überlegungen ergeben.

Das System ist ein heterogenes. $\left.{ }^{1}\right)$ Der Vorgang, welcher in Aufnahme von Wasser besteht, wird mit einer gewissen

1) Unlängst hat Hans Euler in einer Literaturzusammenstellung über die Reaktionsgeschwindigkeit enzymatischer Vorgänge (Katalyse durch Fermente, Diese Zeitschrift, Bd. XLV, S. 421, 1905) die Spaltung einer wässerigen Eigelbemulsion durch den Magensteapsin unter die in echter oder in kolloidaler Lösung verlaufenden Vorgänge eingeordnet und aus den Versuchsdaten einer der darauf bezüglichen Arbeiten (Waldemar Stade, Untersuchungen über das fettspaltende Ferment des Magens, Hofmeister's Beiträge, Bd. III, S. 29) ihre Geschwindigkeitskonstanten nach der für in homogener Lösung verlaufenden monomolekularen Reaktionen geltenden Formel berechnet. Die starke Abnahme der so erhaltenen Konstanten hat Euler damit erklärt, daß die bei der Spaltung entstandenen Säuren das Enzym schädigen. (Kastle und Loewenhart (l. c.) haben nämlich gefunden, daß bei der Spaltung von Äthylbutyrat die abgespaltene Buttersäure die Wirksamkeit des Enzyms herabsetzt.) Ich bin mit Euler's Auffassung dieser Sachen nicht einverstanden aus folgenden Gründen: Erstens ist nicht einzusehen, daß in Wasser sehr schwer lösliche bezw. nur quellende Stoffe, wie die Fette und Lecithine es sind, bloß dadurch, wenn auch nur kolloidal gelöst sein sollen, weil sie mit einem Eiweißstoffe, wie es im Eigelb der Fall ist, zusammen anwesend sind. Zweitens aus den von Gobley und Loebisch ausgeführten Analysen des Eigelbs, welche ich hierher setze (vgl. J. König, Chemie der menschlichen Nahrungs- und Genußmittel, 4. Aufl., Bd. II, S. 575, Berlin 1904):

$\begin{array}{lc}\text { Wasser } & 51,8 \\ \text { Vittelin } & 15,8 \\ \text { Nuclein } & 1,5 \\ \text { Palmitin, Stearin, Olein } & 20,3 \text { bezw. 23,2 } \\ \text { Cholesterin } & 0,4 \\ \text { Glycerinphosphorsäure } & 1,2 \\ \text { Cerebrin } & 0,3 \\ \text { Farbstoffe } & 0,5 \\ \text { Salze } & 1,0 \text { bezw. 1,5 }\end{array}$

geht hervor, daß in demselben nicht die Mono-, sondern die Triglyceride der verschiedenen Fettsäuren enthalten sind. Wären somit die zu spaltenden Fette, im Gegensatz zu meiner Ansicht, wirklich gelöst, so dürfte zur Berechnung der Reaktionsgeschwindigkeit doch nicht die für monomolekulare Reaktionen geltende Formel angewendet werden, sondern es müßten Formeln entwickelt werden, welche dem stufenweisen 
Geschwindigkeit in der Wasserphase verlaufen. Die in der Zeiteinheit zersetzte Menge wird (so lange Wasser im Vergleich zum Fett in großem Überschuß vorhanden ist), nur von der Konzentration des jeweils unzersetzten Fettes in der Wasserphase abhängen. Das Maximum dieser Konzentration bei einer bestimmten Temperatur ist durch die Löslichkeit des Fettes in Wasser, d. h. durch das Gleichgewicht zwischen gelöstem und ungelöstem Fett gegeben. Wird bei der Spaltung das - durch Entfernen von etwas Fett aus der Lösung - gestörte Gleich-

Verlauf der Spaltung der verschiedenen Fette Rechnung tragen, wie es Osc. Knoblauch für die Darstellung der Reaktionsgeschwindigkeit der Spaltung des Bernsteinsäurediäthylesters abgeleitet hat. Drittens können bei der Spaltung - was sich auch aus der Analyse des Eigelbes ergibt - nur die in Wasser fast unlöslichen, hochmolekularen Fettsäuren entstehen. Daß deren sehr geringer in Lösung anwesender Anteil das. Steapsin ebenso schädigen sollte, wie in Kastle und Loewenhart's Versuchen die leichtlösliche, somit auch in grösserer Konzentration anwesende Buttersäure, ist nicht wahrscheinlich.

Es läßt sich überhaupt die Frage nicht unterdrücken, ob die mathematische Behandlung vieler enzymatischer Vorgänge auf Grundlage des vorhandenen Versuchsmaterials überhaupt statthaft ist. Abgesehen davon, daß bis jetzt kaum ein Enzym in einigermaßen reinem Zustand bekannt ist und daß oft mehrere Enzyme zusammen vorkommen, die sich in ihrer gegenseitigen Wirksamkeit sehr wohl beeinflussen können, ist es schwer verständlich, daß, während die mathematische Darstellung wohlbekannter und verhältnismäßig einfacher chemischer Vorgänge wie z. B. die eben erwähnte Spaltung der Ester der mehrbasischen Säuren eine ist - schon recht verwickelte Formeln erfordert, so sehr komplizierte und so wenig bekannte Vorgänge, wie z. B. die peptische Verdauung des Eiweißes ist, durch so einfache Formeln vollständig darstellbar sein sollten, wie sie sich z. B. aus Sjöqvist's (Skand. Arch., Bd. V, S. 277, 1894) Versuchen ergeben haben.

Die Verhältnisse auf diesem Gebiete erinnern an jene, die vor etwa einem Jahrzehnt bei der Anwendung der allgemeinen Ghemie auf andere Gebiete der physiologischen Chemie wahrnehmbar waren.

Obzwar Stoffe wie Eiweiß, Albumose, Pepsin (!) damals verhältnismäßig wenig bekannt waren, fanden Bugarszky und Liebermann (Pflüger's Archiv, Bd. LXXII, S. 51) unter Anwendung der exaktesten Versuchsanordnungen, welche eigentlich genau definierte Stoffe voraussetzen, daß 1 Mol. Eiweiß (abgerundet) \& Mol. Salzsäure bezw. Natronlauge; 1 Mol. «Albumose» 3 Mol. Salzsäure bezw. Natronlauge; $2 \mathrm{Mol}$. Pepsin 1 Mol. Salzsäure binden können. Heute, wo unsere Kenntnisse 
gewicht durch in Lösung Gehen der entsprechenden Fettmenge so schnell wieder hergestellt, als seine Störung erfolgt, so wird die Konzentration des jeweils unzersetzten Fettes, somit auch dessen in der Zeiteinheit zersetzte Menge konstant bleiben, die Geschwindigkeitsformel $\mathrm{KC}=\frac{\mathrm{x}}{\boldsymbol{y}}$ (worin $\mathrm{x}$ die zur Zeit $\boldsymbol{9}$ zersetzte Menge, $\mathrm{C}$ die Löslichkeit des Fettes in Wasser bedeutet) ${ }^{1}$ ) den Vorgang richtig wiedergeben. ${ }^{2}$ )

in der Eiweißchemie entsprechend vertieft sind, hat sich aus der durch Walter Neumann erfolgten physikalisch-chemischen Bearbeitung von Siegfried's Peptonen (Diese Zeitschrift, Bd. XLV, S. 216-252) nicht mit unzweifelhafter Sicherheit ergeben, ob diese Stoffe zwei- bezw. dreibasische Säuren und ein- bezw. zweisäurige Basen sind, oder etwa als sechsbezw. vierbasische Säuren und als vier- bezw. zweisäurige Basen anzusehen wären.

In den neunziger Jahren fand Sabanejeff durch Gefrierpunktsbestimmungen, daß das Molekulargewicht des Glykogens 1620 ist (vgl. die Theoretische Chemie von Nernst, 4. Aufl., S. 411) und vor kurzer Zeit fand Frau Gatin-Gružewska (Pflüger's Arch., Bd. CIII, S. 282) durch Wiederholung der Depressionsbestimmungen mit dem von ihr dargestellten, reinen Glykogen, daß sein Molekulargewicht nicht zu ermitteln ist, denn entweder ist das Glykogen löslich und dann ist sein Molekulargewicht ungemein groß, oder es ist unlöslich in Wasser und dann kann sein Molekulargewicht beliebig groß sein. Unter der Annahme der Löslichkeit wäre als die untere Grenze für das Molekulargewicht 140000 anzunehmen, also etwa $86 \mathrm{mal}$ so viel, als früher gefunden worden ist. (Dieses Resultat ist soeben durch E. v. Knaffl-Lenz, Diese Zeitschrift, Bd. XLVI, Heft 3, auf rein chemischem Wege bestätigt worden.)

Wenn man sich den enormen Fortschritt vergegenwärtigt, welcher in manchen Teilen der Chemie durch zielbewußtes Anwenden physikalischchemischer Methoden erzielt worden ist, so darf man eben nicht vergessen, wie hochentwickelt die Kenntnisse der quantitativen Verhältnisse auf gerade diesen Gebieten bereits gewesen sind, als man sie vom Standpunkte der neuen Lehren zu bearbeiten begann!

1) Richard Löwenherz, Übèr die Verseifungsgeschwindigkeit einiger Ester. Zeitschrift für physik. Chemie, Bd. XV, S. 389, 1894.

2) $\mathrm{DaB}$ eigentlich mehrere Fette gleichzeitig hydrolysiert werden und daß die Hydrolyse eines jeden in Stufen erfolgt, ist bei dieser Entwickelung nicht berücksichtigt, denn diese Unterschiede werden wegen der äußerst geringen Löslichkeit der Fette nur in der allerersten Zeit zur Geltung kommen. 
Da aus der ausgedehnt studierten ${ }^{1}$ ) Hydrolyse der Ester durch die Gegenwart von Säuren als Katalysatoren folgernd, es als ziemlich sicher anzunehmen ist, daß auch die Hydrolyse der Glycerinester der hochmolekularen Fettsäuren in Gegenwart von Enzymen ein nicht allzu rasch verlaufender Vorgang ist, andererseits durch Darstellung einer Emulsion eine große Oberfläche geschaffen wird, dürfte es anzunehmen sein, daß wenigstens für den Fall der Verwendung kleiner, oder wenig wirksamer Enzymmengen - für ein genügend schnelles Ersetzen des durch die Spaltung fortgeschafften Fettes gesorgt ist. $\mathrm{Da}$ die Formel auch für diesen Fall nicht zutrifft (die oben mitgeteilte Tabelle ist mit einer trägen Steapsinlösung erhalten), so wäre ein reversibles oder ein irreversibles Unwirksamwerden des Enzyms bei der Hydrolyse als eine vorläufige Erklärung nicht von der Hand zu weisen, wenn nicht in der Literatur folgende Versuchsergebnisse vorhanden wären.

Im Fliegenpilz (Amanita muscaria L.) ist ein zwar langsam, aber intensiv fettspaltendes Enzym vorhanden. Julius Zellner, ${ }^{2}$ ) der dasselbe aufgefunden hat, teilt auch mehrere Tabellen über den zeitlichen Verlauf der damit bewirkten Spaltung mit. In diesen sind die Kubikzentimeter n/2-Natronlauge angegeben, welche verschiedene gewogene Ölmengen zur Neutralisation bedurften, nachdem das Enzym eine gewisse Stundenzahl auf sie eingewirkt hatte. Ich habe diese Zahlen

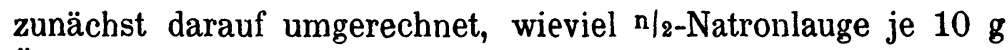
Öl zu den angeführten Zeiten verbraucht haben, sodann die Konstanten nach der Formel $\frac{x}{y}$ bezw. $\frac{x}{\sqrt{y}}$ ausgerechnet und teile sie hierfolgend mit.

In allen Versuchen wurde mit je $40 \mathrm{~g}$ entfettetem Pilzpulver gearbeitet. In Tabelle A und B wurde damit je $40 \mathrm{~g}$ Olivenöl, in Tabelle $\mathrm{C}$ Talg gespalten; in Tabelle A in Gegenwart von $10 \mathrm{~g}$ Wasser, in Tabelle B und $\mathrm{G}$ in Gegenwart von Bd. XIV.

1) Löwenherz (l. c.), de Hemptinne, Ztschr. f. physikal. Chem.,

2) Zur Chemie des Fliegenpilzes (Amanita muscaria L.), II. Mitteilung, Monatshefte f. Chem., Bd. XXVI, 727 ff., 1905. 
$10 \mathrm{~g} \mathrm{n} / 4$-Schwefelsäure. Tabelle $\mathrm{A}$ und $\mathrm{B}$ waren bei $20^{\circ}$, Tabelle C bei $45^{\circ}$ erhalten worden. Die Buchstaben $\frac{x}{y}, \frac{x}{\sqrt{9}}$ haben dieselbe Bedeutung wie früher, $\mathrm{x}$ bedeutet jedoch Kubikzentimeter $\mathrm{n} / \mathrm{z}$-Natronlauge und $\boldsymbol{\vartheta}$ Stunden. Die Konstanten sind somit 12 mal größer als in den anderen Tabellen.

\begin{tabular}{|c|c|c|c|}
\hline \multirow[t]{2}{*}{$\vartheta$} & $\mathbf{x}$ & $\frac{x}{y}$ & $\frac{x}{\sqrt{\sqrt{\vartheta}}}$ \\
\hline & \multicolumn{3}{|c|}{ A. } \\
\hline 160 & 8,84 & 0,055 & 0,70 \\
\hline 304 & 19,95 & 0,066 & 1,14 \\
\hline 472 & 28,05 & 0,060 & 1,29 \\
\hline \multirow{2}{*}{\multicolumn{4}{|c|}{ B. }} \\
\hline & & & \\
\hline 48 & 2,80 & 0,058 & 0,40 \\
\hline 111 & 7,37 & 0,066 & 0,70 \\
\hline 304 & 17,80 & 0,059 & 1,02 \\
\hline 497 & 23,62 & 0,048 & 1,06 \\
\hline \multicolumn{4}{|c|}{ 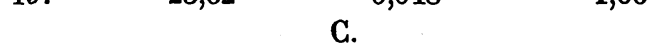 } \\
\hline 137 & 12,30 & 0,09 & 1,05 \\
\hline 304 & 20,70 & 0,068 & 1,19 \\
\hline 646 & 29,70 & 0,046 & 1,17 \\
\hline
\end{tabular}

Während sich aus Tabelle $\mathrm{A}$ und $\mathrm{B}$ die annähernde Konstanz der theoretischen Formel ergibt, geht aus Tabelle C. die Konstanz der experimentell gefundenen hervor. Dabei ist der Unterschied in den Versuchsreihen nur der, daß in den ersten zwei Olivenöl, im dritten Talg hydrolysiert worden ist. Der Einwand, daß die Lösung nicht immer in bezug auf Fett gesättigt gewesen wäre, kann in diesem Fall wegen des sehr langsamen Verlaufs der Spaltung nicht gemacht werden. Der Vorgang wird durch einen vorläufig nicht ersichtlichen «Nebenumstand" wesentlich beeinflußt, so daß es nicht so sehr unwahrscheinlich ist, daß weitere Variierungen der Versuchsanordnungen auch mit Pankreassteapsin andere, der theoretischen Forderung entsprechende Ergebnisse haben können. Ich beabsichtige die Frage weiter zu verfolgen.

Leipzig, Chemische Abteilung des Physiolog. Instituts, November 1905. 